\title{
New Fuzzy Integral for the Unit Maneuver in RTS Game
}

\author{
Peter Hiu Fung Ng, YingJie Li, and Simon Chi Keung Shiu \\ Department of Computing, The Hong Kong Polytechnic University, Hong Kong \\ \{cshfng, csyjli, csckshiu\}@comp.polyu.edu.hk
}

\begin{abstract}
A new strategy planning is proposed to improve the effectiveness of unit maneuver in RTS game. Following several other researchers, our approach is based on the technique of potential field to provide an efficient path searching in dynamic environment. However, we adopt the Fuzzy Measure and Integral to indicate the interaction among the unit types. Hence, we also propose a new Fuzzy Integral to improve the quality of unit maneuver. Diversion and flank attack can be planned in potential field dynamically. We implement and present our techniques on real RTS game platform. The result is promising in this complex environment.
\end{abstract}

Keywords: Fuzzy Measure, Fuzzy Integral, Potential Field, CMA-ES, RTS.

\section{Introduction}

Unit formation and target of attack is the cores of unit maneuver in real time strategy (RTS) game. It is complicated as it consists of a great quantity of possibilities. Multiple targets and the intransitive superiority of unit formation lead the unit maneuver to remain a problem. Traditional tree searching or $\mathrm{A}^{*}$ searching is unable to handle these two properties. There are too many weightings and each of them will interact with the others. In this research, we apply potential field, Fuzzy Measure and Integral to solve this problem. Potential field is suitable for complicated and various environment with multiple targets. However, it does not consider non-additive property. We integrate it with Fuzzy Measure and Integral to extend simple additive property to non-additive property. It provides the ability to handle interaction among different targets. We also propose a new Fuzzy Integral, Directional based Fuzzy Integral, to support the flank and diversion attack in unit formation planning.

\section{$2 \quad$ Literature Review}

More and more researchers are interested in RTS game and have recently generated many important outputs in the community $[1,2,3]$. They shared the same view on RTS game environment. It is hostile and dynamic. Counter maneuvers need to be implemented under uncertainty and time pressure. The classic potential field consists of two virtual forces $[4,5]$. One is attractive potential force which is used to represent the goal. Another force is repulsive potential force which is used to represent the obstacle. Multi-agents potential fields of Hagelback [6] showed the importance to unit maneuver and tactics development. 
Fuzzy Measure and Integral have been proven the ability of interaction handling $[7,8]$. We have also implemented Fuzzy Integral to evaluate the combined power of units $[9,10,11]$. It is an efficient way to handle the interaction problem in RTS game. However, the aggregator of Fuzzy Integral has not been investigated and visualized very well. Our proposed idea here is to measure the opponent power by the Fuzzy Integral and provide a planning model for unit maneuver.

\section{Methodology}

The main ideas of this model are to minimum the interaction of enemy units and maximize the interaction of player units. First, the weighting of Fuzzy Measure for different unit types are learnt from real RTS game data. Then, the weightings are assigned to a potential field. All potentials of different unit types are summed up with geometric functions. Finally, the highest potential will become the next movement target.

\subsection{Learning Fuzzy Measure from Real RTS Game Data}

We use the Warcraft III replay to learn the interaction from real data [6]. For each replay $r$, unit proportions and scores of player and enemy are extracted. $X=\left\{x_{1}, x_{2}\right.$, $\left.\ldots, x_{n}\right\}$ is used to represent the enemy unit proportion, where $x_{i}$ is the proportion of unit type $i$ and $n$ is the total number of unit type. $Y=\left\{y_{1}, y_{2}, \ldots, y_{n}\right\}$ is used to represent the player unit proportion. These quantities here are measured by the amount of resources used instead of the physical quantity count. Then it is normalized by a normalization function $f$.The scores, $\operatorname{Score}_{\text {player }}(r)$ and $\operatorname{Score}_{\text {enemy }}(r)$ are also extracted from each replay $r$.

Fuzzy Measure is used to represent the combined power of enemy and player. Fuzzy measure of enemy is defined as a mapping: $\mu: P(X) \rightarrow[0,1]$, where $P(X)$ is the power set of $X$, i.e., all the $\left(2^{\mathrm{n}}-1\right)$ subsets of $X$. The Fuzzy Measure of player is defined as a mapping: $\mu: P(Y) \rightarrow[0,1]$ with the same condition. $\mu(X)$ and $\mu(Y)$ are trained separately by CMA-ES and Fuzzy Integral. First, the $\mu(X)$ or $\mu(Y)$ are randomly assigned. Then the Fuzzy Integral, $\operatorname{Score}_{\text {player }}(r)$ and $\operatorname{Score}_{\text {enemy }}(r)$ are used to be the fitness function. The solution is optimized throughout the iteration. Detail procedures are shown in our previous researches $[9,10,11]$.

\subsection{Combining Choquet Integral and Potential Field}

The contribution of each unit type in CI should be investigated and defined as a point of interest (POI) in the potential field. CI is converted into another form as shown in Equation (1). Assume $a_{i}$ is the sorted $x_{i}$. $C\left(a_{i}\right)$ is used to represent the contribution of unit type $a_{i}$. Its equation is shown in Equation (2). As the Fuzzy Measure is nonmonotonic, the contribution of unit type, $C\left(a_{i}\right)$, may be negative. For example, if $\mu\left(a_{2}, a_{3}\right)<\mu\left(a_{3}\right)$, then $C\left(a_{2}\right)$ will be negative. Therefore, the negative interaction of enemy could be identified.

$$
\begin{gathered}
\sum_{i=1}^{n}\left(a_{i}\right) \cdot\left[\mu\left(x \mid f(x) \geq a_{i}\right)-\mu\left(x \mid f(x) \geq a_{i+1}\right)\right] \\
C\left(a_{i}\right)=\left(a_{i}\right) \times\left[\mu\left(x \mid f(x) \geq a_{i}\right)-\mu\left(x \mid f(x) \geq a_{i+1}\right)\right]
\end{gathered}
$$




\subsection{New Integral for Potential Field}

Although the Choquet Integral could present the positive and negative interaction among the unit type, the priory sorting of $f(x)$ is difficult for the game developer to understand. Hence, the meaning of $C\left(a_{i}\right)$ which is related to the subset of $a_{i}$ and its upper term $a_{i-1}$ is also difficult to understand. The individual contribution of $a_{i}$ and the interaction to the other units are mixed together. Therefore, we develop Directional based Fuzzy Integral (Directional based FI). It is used to describe the correlation of two unit types. The entire equation is shown in Equation (3). The integral is divided into two parts. The first part, $O$, is its individual contribution. The second part, $I$, is used to describe all the correlation of unit types.

$$
\begin{array}{r}
(d) \int f(x) d \mu=\sum_{i=1}^{n}(O+I) \\
\text { where } O=f\left(x_{i}\right) \mu\left(x_{i}\right) \\
I=w \sum_{j=1, j \neq i}^{n} f\left(x_{i}\right)\left[\mu\left(x_{i}, x_{j}\right)-\mu\left(x_{j}\right)\right] \\
f\left(x_{i}\right) \neq 0, f\left(x_{j}\right) \neq 0
\end{array}
$$

A potential field with size $S \times S$ is generated. The equation of each point in potential field, $P(x, y)$, is shown in the Equation (4). Each unit type is a point of interest (POI). The unit type's coordinate, $P_{i}$, is extracted in the battle. By the Equation (3), the individual contribution and interaction can be indicated independently. Then, they are combined with the decay function, $\varphi$ which is a form of Euclidean distance. $D$ is a weighting to control area of the affected. The larger the $D$, the smaller affected area.

$$
P(\mathrm{x}, \mathrm{y})=\left\{\begin{array}{cc}
\left.\sum_{i=1}^{n}\left(O \times \varphi\left(P(\mathrm{x}, \mathrm{y}), P_{i}\right)\right)+I \times \varphi\left(P_{i}, P_{j}\right)\right) & (4.1),(4.2) \text { is true } \\
\sum_{i=1}^{n}\left(O \times \varphi\left(P(\mathrm{x}, \mathrm{y}), P_{i}\right)\right) & \text { Otherwise }
\end{array}\right.
$$

where $n$ is the total number of unit type, $P_{i}$ is the coordinate of $a_{i}$

$$
\begin{gathered}
\varphi\left(P(\mathrm{x}, \mathrm{y}), P_{i}\right)=\left(D \times \log \left(\sqrt{\left[P(\mathrm{x})-P_{i}(\mathrm{x})\right]^{2}+\left[P(\mathrm{y})-P_{i}(\mathrm{y})\right]^{2}}\right)\right)^{-1} \\
\varphi\left(P_{i}, P_{j}\right)=\left(D \times \log \left(\sqrt{\left[P_{i}(\mathrm{x})-P_{j}(\mathrm{x})\right]^{2}+\left[P_{i}(\mathrm{y})-P_{j}(\mathrm{y})\right]^{2}}\right)\right)^{-1}
\end{gathered}
$$

$D$ is the weighting to control area of affected

$$
\begin{gathered}
\left.\left.\sqrt{\left[P(\mathrm{x})-P_{j}(\mathrm{x})\right]^{2}+\left[P(\mathrm{y})-P_{j}(\mathrm{y})\right]^{2}}\right) \leq \sqrt{\left[P_{i}(\mathrm{x})-P_{j}(\mathrm{x})\right]^{2}+\left[P_{i}(\mathrm{y})-P_{j}(\mathrm{y})\right]^{2}}\right) \\
\theta\left(P_{i}, P_{j}\right)-\alpha \leq \theta\left(P(x, y), P_{i}\right) \leq \theta\left(P_{i}, P_{j}\right)+\alpha \\
\text { where } \theta\left(P_{1}, P_{2}\right)=\arctan \left(\frac{P_{1}(y)-P_{2}(y)}{P_{1}(x)-P_{2}(x)}\right) \text { and } \alpha=\pi / 4
\end{gathered}
$$


The affected area of individual contribution, $O$, is presented as a circle which is similar to the original potential field. However the affected area of interaction part, $I$, is presented as a sector of circle and points to another unit. The interaction part is generated in the area in between two points as the expression (4.1) and within a degree, $\alpha$, as the expression (4.2).

The potential, $I$, represents the contribution of $x_{i}$ to $x_{j} . \varphi\left(P_{i}, P_{j}\right)$ is a decay function which is used to enlarge the potential if the enemy unit is closer to each other. Figure 1(a) show the combined potential of two unit types. If $P_{1}$ and $P_{2}$ are getting away, the potential will drop sharply as shown in Figure 1(b).

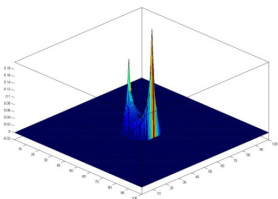

(a)

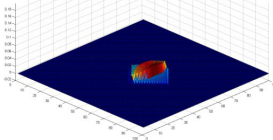

(b)

Fig. 1. Effect of the decay function, $\varphi\left(P_{i}, P_{j}\right)$

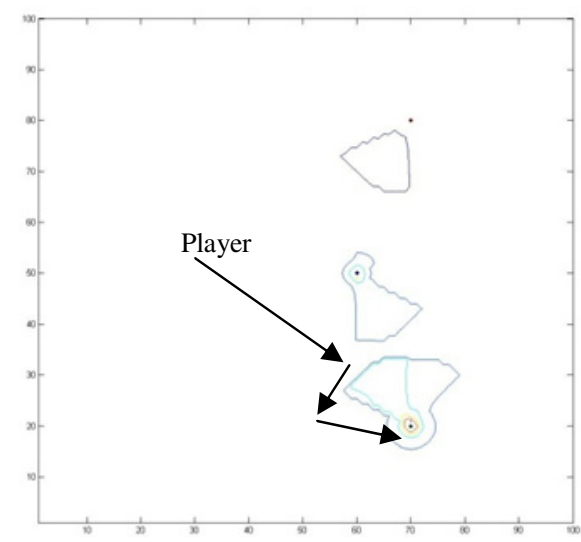

(a)

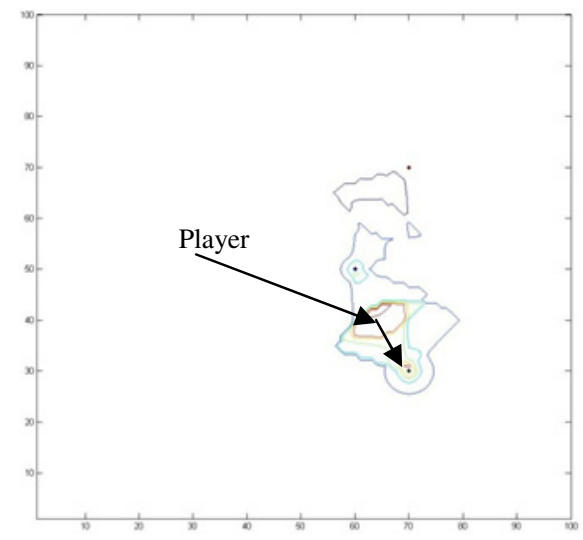

(b)

Fig. 2. Effect of the decay function, $\varphi\left(P_{i}, P_{j}\right)$

\subsection{Flanking and Diversion Attack}

After the enemy and player potential field are generated, scripting is needed to decide the action of each unit type, such as flanking, diversion, direct attack or cooperation. First the highest potential of enemy and player are compared. One of them is selected. If the potential of player is higher, cooperation is performed to maintain the unit formation. On the other hand, if the potential of enemy is higher, the player will perform the flank (Figure 2(a)) or diversion attack (Figure 2(b)). The highest potential will 
become the destination and the path is generated. All the process is updated at certain time slot. Destination and path is continually updated.

\section{$4 \quad$ Experimental Result and Discussion}

To prove the performance of potential field and fuzzy integral, we used Warcraft III to simulate the experiment. 1553 replays are collected from official competitions and used for training the Fuzzy Measure.

For CMA-ES, 50 search points are generated randomly. Their fitness is computed by Fuzzy Integral and compared with the scores of real game data. 25 better solutions are selected to computer mean vector, step size and covariance matrix. The search points are updated by these three parameters for the next generation. After 2500 iteration, the Fuzzy Measures will be used for testing.

A battle field is generated. Three unit types are randomly selected for each side. The number of unit in each type is randomly assigned but the total amount of the army is fixed to 20. Three unit maneuver models are tested. The first one is rule based system of Warcraft III. The second one is potential field and Choquet Integral. The last one is potential field and Directional based FI. For each experiment, 50 battles are preformed. The result has been stated in Table 1. The winning percentage of potential field with Directional based fuzzy integral is the highest. Compared with rule based system, potential field with Choquet Integral has a $29 \%$ improvement and the potential field with Directional based fuzzy integral has a $48 \%$ improvement.

The performance of unit maneuver is optimized. By observing the battle, potential fields with Directional based fuzzy integral showed a significant improvement when flank and diversion attack can be performed.

Table 1. Comparison of different Unit Maneuver

\begin{tabular}{lc}
\hline \hline Unit Maneuver & Wining \\
\hline Rule based System of Warcraft III & $42 \%$ \\
Potential field and Choquet Integral & $54 \%$ \\
Potential field and Directional based fuzzy Integral & $62 \%$ \\
\hline \hline
\end{tabular}

\section{Conclusion}

In this paper, we have developed an adversarial real time planning model for unit maneuver in RTS game. We have extended the normal additive properties to nonlinear for potential field. We also defined a new type of fuzzy integral called Directional-based FI. The performance of path finding is optimized. Details unit maneuver, such as flank and diversion attack can be performed. The future work will focus on extending the result of feature interaction to perform more advance unit maneuver in RTS game, such as tracking problem. 


\section{References}

1. Buro, M.: Call for AI research in RTS games. In: Proceedings of the AAAI 2004 Workshop on Challenges in Game AI, pp. 139-142 (2004)

2. Lucas, S.M.: Computational Intelligence and AI in Games. IEEE Transaction on Computational Intelligence and AI in Games 1, 1-3 (2009)

3. Alexander, N.: Game AI is Dead. Long Live Game AI! IEEE Intelligent Systems 22(1), 9-11 (2007)

4. Weijun, S., Rui, M., Chongchong, Y.: A Study on Soccer Robot Path Planning with Fuzzy Artificial Potential Field. In: 2010 International conference on Computing, Control and Industrial Engineering, pp. 386-390 (2010)

5. Yin, L., Yin, Y.: An Improved Potential Field Method for Mobile Robot Path Planning in Dynamic Environment. Proceeding of the 7th World Congress on Interlligent Control and Automation, 4847-4852 (2008)

6. Hagelback, J., Johansson, S.: Using multi-agent potential fields in real-time strategy games. In: Proceedings of the 7th International Joint Conference on Autonomous Agents and Multiagent Systems, vol. 2, pp. 631-638 (2008)

7. Ishii, K., Sugeno, M.: A model of human evaluation process using fuzzy measure. Int. J. Man-Machine Studies 22, 19-38 (1985)

8. Murofushi, T., Sugeno, M.: The Choquet integral in multiattribute decision making. Fuzzy Measures and Integrals: Theory and Applications, 333-347 (2000)

9. Li, Y.J.: Apply Different Fuzzy Integrals in Unit Selection Problem of Real Time Strategy Game. In: Proceeding of 2011 IEEE International Conference on Fuzzy System (FuzzIEEE 2011), Taipei, Taiwan, June 27-30, pp. 170-177 (2011)

10. Ng, P.H.F., Li, Y.J., Wang, H.B., Shiu, S.C.K.: Bottom-Up Strategy Planning Model by applying Fuzzy Integral in RTS Game. In: Proceeding of Joint 5th In-ternational Conference on Soft Computing and Intelligent Systems and 11th International Symposium on Advanced Intelligent Systems (SCIS \& ISIS), Okayama, Japan, December 8-12, pp. 1579-1584 (2010); Best Student Paper Award

11. Li, Y.J., Ng, P.H.F., Wang, H.B., Li, Y., Shiu, S.C.K.: Applying Fuzzy Integral for Performance Evaluation in Real Time Strategy Game. In: Proceeding of 2010 2nd International Conference on Information and Multimedia Technology (ICIMT), Hong Kong, RPC China, December 28-30, pp. 168-172 (2010) 